\title{
AFM and electroanalytical studies of synthetic oligonucleotide hybridization
}

\author{
A.-M. Chiorcea Paquim ${ }^{\text {a }}$, V.C. Diculescu ${ }^{\text {a }}$, T.S. Oretskaya ${ }^{\text {b }}$, A.M. Oliveira Brett ${ }^{\text {a } *}$ \\ ${ }^{a}$ Departamento de Química, Faculdade de Ciências e Tecnologia, Universidade de Coimbra, 3004-535 Coimbra, Portugal \\ ${ }^{\mathrm{b}}$ Chemistry Department, Moscow State University, Vorobjovy Gory, 119899, Moscow, Russia
}

Available online 28 July 2004

\begin{abstract}
The first and most important step in the development and manufacture of a sensitive DNA-biosensor for hybridization detection is the immobilization procedure of the nucleic acid probe on the transducer surface, maintaining its mobility and conformational flexibility. MAC Mode AFM images were used to demonstrate that oligonucleotide (ODN) molecules adsorb spontaneously at the electrode surface. After adsorption, the ODN layers were formed by molecules with restricted mobility, as well as by superposed molecules, which can lead to reduced hybridization efficiency. The images also showed the existence of pores in the adsorbed ODN film that revealed large parts of the electrode surface, and enabled non-specific adsorption of other ODNs on the uncovered areas. Electrostatic immobilization onto a clean glassy carbon electrode surface was followed by hybridization with complementary sequences and by control experiments with non-complementary sequences, studied using differential pulse voltammetry. The data obtained showed that non-specific adsorption strongly influenced the results, which depended on the sequence of the ODNs. In order to reduce the contribution of non-specific adsorbed ODNs during hybridization experiments, the carbon electrode surface was modified. After modification, the AFM images showed an electrode completely covered by the ODN probe film, which prevented the undesirable binding of target ODN molecules to the electrode surface. The changes of interfacial capacitance that took place after hybridization or control experiments showed the formation of a mixed multilayer that strongly depended on the local environment of the immobilized ODN.
\end{abstract}

(C) 2004 Elsevier B.V. All rights reserved.

Keywords: DNA; DNA-biosensor; Hybridization; Adsorption; Non-specific adsorption; AFM

\section{Introduction}

DNA sequences are unique to each organism and any self-replicating biological organism can be discriminated using DNA hybridization. The need for the analysis of gene sequences and monitoring microorganisms in medical, environmental or food control, the determination of the oxidative damage to DNA and understanding of DNA interactions with molecules or ions led to the development of DNA biosensors.

Great progress has been made in developing small, portable and viable DNA-based biosensors, which are of enormous interest due to their numerous potential applications, to detect chemical compounds by electrical (Chiti et al., 2001; Oliveira Brett et al., 1999; Oliveira Brett, 2004;

\footnotetext{
* Corresponding author. Tel.: +351-239-835295; fax: +351-239-835295

E-mail address: brett@ci.uc.pt (A.M. Oliveira Brett).
}

Palecek et al., 1998, 2002; Pedano and Rivas, 2003; Strasak et al., 2002; Vetterl et al., 2000; Wang, 2000, 2002; Wang et al., 2002) thermal (Breslauer et al., 1986; Ratilainen et al., 1998), mechanical (Ito et al., 1996; Nicolini et al., 1997; Su and Thompson, 1995) or optical (Kleinjung et al., 1997; Piunno et al., 1995; Waterson et al., 2002a) signal transduction.

Nucleic acid based biosensors normally employ immobilized DNA sequences on the sensor surface as the recognition element and sequence specific hybridization can be monitored and analyzed. Short, synthetic oligonucleotides (ODNs) are progressively replacing genomic and cloned DNA and are ideal chemical recognition elements, because the hybridization is highly sequence-selective.

The hybridization strategy at a DNA-biosensor consists of three steps: immobilization of the probe ODN at the transducer surface, hybridization with the complementary strand (target) present in the solution and transduction. The DNA immobilization procedure on the electrode surface is a very 
important aspect since it influences the characteristics of the DNA probe, the sensor response and its performance. Several different DNA adsorption methods have been used on different types of electrodes (Pividori et al., 2000; Yang et al., 1997). The specific interaction of DNA molecules with the electrode surface, the conformations that DNA can adopt during immobilization and the degree of surface coverage are still not well understood despite the extensive use of DNA biosensors. Careful attention has been paid to different factors such as substrate material, DNA nature, immobilization density, a variety of experimental conditions such as temperature, $\mathrm{pH}$ or ionic strength, and sample processing, in order to increase the selectivity, sensitivity or final speed of the hybridization assay (Waterson et al., 2002b).

Magnetic AC mode atomic force microscopy (MAC Mode AFM) is a gentle technique that allows the investigation of DNA molecules that are loosely attached to conducting electrode surfaces (Oliveira Brett and Chiorcea, 2003). MAC Mode AFM was used to study the free adsorption and adsorption under applied potential of short synthetic ODNs onto a highly oriented pyrolytic graphite (HOPG) electrode surface. The overall topography of the DNA based biosensor surface obtained by adsorption of ODNs on the electrode surface was investigated, in order to differentiate between the biosensor efficiency and the extent of surface coverage of the electrode when different ODN sequences were adsorbed. An HOPG electrode was used as substrate, because it is extremely smooth, inert in air and has easy to clean terraces on its basal plane, which represents an important requirement for imaging biological molecules. Comparatively, for a $1000 \mathrm{~nm} \times 1000 \mathrm{~nm}$ surface area, the glassy carbon has a root-mean-square (r.m.s.) roughness of $2.10 \mathrm{~nm}$, while the HOPG electrode surface has a r.m.s. roughness of $<0.06 \mathrm{~nm}$ (Oliveira Brett and Chiorcea, 2003, 2004).

In this paper, the hybridization of two complementary ODNs without the use of external factors is reported. The experiments were carried out at a clean and at an ODN-modified glassy carbon electrode. The hybridization event was recognized using differential pulse voltammetry, via the appearance of guanosine and adenosine oxidation peaks. Electrochemical impedance spectroscopy was also used to assess the changes that took place in the electrode interfacial region. Control experiments using non-complementary ODNs were performed in order to characterize the non-specific adsorption that took place during the hybridization experiments.

\section{Experimental}

\subsection{Materials}

The 10-mer ODNs used in this study were synthesized on an Applied Biosystems 380B automated DNA synthesizer (USA) using reagents for ODN chemistry purchased from Fluka (Germany). The purity of the ODN sequences was verified by NMR and HPLC analysis. The base sequences used were:

\begin{tabular}{|c|c|}
\hline Probe & Oligo(1) $5^{\prime}$-GTAGATCACT-3' \\
\hline $\begin{array}{l}\text { Target-complementary } \\
\text { sequence: }\end{array}$ & Oligo(2) $5^{\prime}$-AGTGATCTAC-3' \\
\hline \multirow{2}{*}{$\begin{array}{l}\text { Control-non- } \\
\text { complementary } \\
\text { sequence: }\end{array}$} & Oligo(3) $5^{\prime}$-AAAAAAAAAA- $3^{\prime}$ \\
\hline & $\begin{array}{ll}\text { Oligo(4) } & 5^{\prime} \text {-AAAGAAAAAG-3' } \\
\text { Oligo(5) } & 5^{\prime} \text {-AAAAGGAGAG-3' } \\
\text { Oligo(6) } & 5^{\prime} \text {-GGGGCCCGGG-3' } \\
\text { Oligo(7) } & 5^{\prime} \text {-CTTTTTCTTT-3' }\end{array}$ \\
\hline
\end{tabular}

The supporting electrolyte was $\mathrm{pH} 4.50 .1 \mathrm{M}$ acetate buffer solution and was prepared using analytical grade reagents and purified water from a Millipore Milli-Q system (conductivity $\left.<0.1 \mu \mathrm{S} \mathrm{cm}^{-1}\right)$. All ODN solutions had a concentration of $0.3 \mu \mathrm{M}\left(\sim 0.9 \mu \mathrm{g} \mathrm{mL}^{-1}\right)$ and were obtained by direct dilution in acetate buffer electrolyte.

Microvolumes were measured using EP-10 and EP-100 Plus Motorized Microliter Pipettes (Rainin Instruments Co. Inc., Woburn, USA). The $\mathrm{pH}$ measurements were carried out with a GLP 21 Crison $\mathrm{pH}$ meter.

\subsection{Atomic force microscopy experimental procedure}

Highly oriented pyrolytic graphite (HOPG), grade ZYH of $15 \mathrm{~mm} \times 15 \mathrm{~mm} \times 2 \mathrm{~mm}$ dimensions, from Advanced Ceramics Co., was used in the AFM study as a substrate. The HOPG was freshly cleaved with adhesive tape prior to each experiment and imaged by MAC Mode AFM in order to establish its cleanliness.

Potential control adsorption was carried out in a one-compartment Teflon cell of $12.5 \mathrm{~mm}$ internal diameter, holding the HOPG substrate, the working electrode, on the base. A Pt wire counter electrode and an Ag wire quasi-reference electrode (AgQRE) were placed in the cell, dipping approximately $5 \mathrm{~mm}$ into the solution. The AgQRE electrode was calibrated against $\mathrm{Ag} / \mathrm{AgCl}$ reference electrode. Electrochemical control was done with a PalmSens potentiostat, running with PalmScan Version 1.11, from Palm Instruments BV, The Netherlands.

AFM was performed with a PicoSPM controlled by a MAC Mode module and interfaced with a PicoScan controller from Molecular Imaging Corp., Tempe, AZ. All the AFM experiments were performed with a CS AFM S scanner with scan range $6 \mu \mathrm{m}$ in $x-y$ and $2 \mu \mathrm{m}$ in $z$, from Molecular Imaging Corporation. Silicon type II MAClevers of $225 \mu \mathrm{m}$ length, $2.8 \mathrm{~N} \mathrm{~m}^{-1}$ spring constants and $60-90 \mathrm{kHz}$ resonant frequencies (Molecular Imaging Co.) were used. All images (256 samples/line $\times 256$ lines) were taken at room temperature; scan rates $1.00-1.13$ lines $\mathrm{s}^{-1}$. The AFM images were processed by flattening in order to remove the background slope and the contrast and brightness were adjusted. Section 
analysis over DNA molecules and films and r.m.s. roughness measurements were performed with PicoScan software Version 4.19, Molecular Imaging Corp.

For MAC Mode AFM surface characterization of the ODN samples prepared by free adsorption, $200 \mu \mathrm{L}$ of the desired ODN solution was placed onto freshly cleaved HOPG surfaces for $3 \mathrm{~min}$. The excess of ODN was gently cleaned with a jet of Millipore Milli-Q water, and the HOPG with adsorbed ODN was then dried in a sterile atmosphere. For ODN samples prepared by adsorption under applied potential, $500 \mu \mathrm{L}$ of the desired ODN solution were placed in the electrochemical cell holding the HOPG working electrode on the base. A positive potential of $+1.0 \mathrm{~V}$ versus AgQRE, was applied to the electrode for $3 \mathrm{~min}$. The HOPG with adsorbed ODN molecules was rinsed with a jet of Millipore Milli-Q water and dried in $\mathrm{N}_{2}$ atmosphere.

\subsection{Electrochemical experiments}

A glassy carbon working electrode $(\mathrm{GCE})(d=1.5 \mathrm{~mm})$, a platinum wire counter electrode and a $\mathrm{Ag} / \mathrm{AgCl}$ saturated $\mathrm{KCl}$ reference electrode were used in a $0.5 \mathrm{~mL}$ one-compartment electrochemical cell. Measurements were carried out using a PGSTAT 10 Autolab Echo-Chemie, Utrecht, The Netherlands. Differential pulse (DP) voltammetry was carried out using GPES software Version 4.9. The experimental conditions for DP voltammetry were: pulse amplitude $50 \mathrm{mV}$, pulse width $70 \mathrm{~ms}$ and scan rate $5 \mathrm{mV} \mathrm{s}^{-1}$. All the voltammograms obtained were smoothed using a Savitsky-Golay algorithm and base line corrected by the moving average method, with a peak width of $1 \mathrm{mV}$, using the GPES software. Electrochemical impedance measurements (EIS) were performed with FRA software Version 4.9. A r.m.s. perturbation of $5 \mathrm{mV}$ was applied over the frequency range $100 \mathrm{kHz}$ to $0.1 \mathrm{~Hz}$ with five frequency values per decade at $+0.5 \mathrm{~V}$ applied potential.

\subsection{Electrochemical experimental procedures}

The electrochemical hybridization experiments at the electrode surface were carried out using a GCE and an ODN-modified GCE surface.

The ODN-GCE surface modification was achieved by applying a potential of $+1.4 \mathrm{~V}$ to a bare GCE whilst immersing it for $2 \mathrm{~min}$ in a solution containing $0.3 \mu \mathrm{M}$ oligo(1).

All experiments were carried out following a four step procedure: probe immobilization, hybridization, transduction and control. During all these steps except for the transduction the solutions were continuously stirred.

\subsubsection{Probe immobilization}

The freshly polished GCE or ODN-modified GCE were placed for $3 \mathrm{~min}$ in a $0.3 \mu \mathrm{M}$ oligo(1) probe solution allowing free adsorption of the ODN to occur. Then the electrode was rinsed with acetate buffer.

\subsubsection{Hybridization}

Hybridization was performed at room temperature by dipping the electrode covered with immobilized oligo(1) probe into a $0.3 \mu \mathrm{M}$ oligo(2) target solution for 3 or $5 \mathrm{~min}$. The electrode was then rinsed with acetate buffer.

\subsubsection{Transduction}

Transduction was carried out in acetate buffer by DP voltammetry and by electrochemical impedance spectroscopy at $+0.5 \mathrm{~V}$ applied potential.

\subsubsection{Control}

Oligo(1) probe immobilization was monitored by dipping the electrode covered with immobilized oligo(1) probe for $5 \mathrm{~min}$ into a solution containing $0.3 \mu \mathrm{M}$ of a non-complementary ODN: oligo(3) till oligo(7). The electrode then was rinsed with acetate buffer and transduction was performed.

\section{Results and discussion}

\subsection{AFM characterization of adsorbed oligonucleotides}

In order to understand the process of adsorption on the electrode surface, the adsorption of the ODN sequences employed in the electrochemical study was first investigated. These ODN-modified surfaces were characterized using MAC Mode AFM in air.

The immobilization of the ODN sequences was achieved by free adsorption during $3 \mathrm{~min}$, as described in the Section 2.

One of the most important steps in detecting DNA hybridization consists in the adsorption of the probe ODN sequence on the carbon electrode surface, in particular the orientation and the packing of the adsorbed ODN probe. Fig. 1A shows a MAC Mode AFM image of the HOPG surface after exposure during $3 \mathrm{~min}$ to the oligo(1) solution, the probe sequence and clearly shows the high capacity of oligo(1) molecules to interact with the HOPG surface. Adsorption was uniform over the surface and the molecules formed small filaments. The r.m.s. roughness of the ODN film in Fig. 1A was $0.47 \mathrm{~nm}$. However, the ODN film was incomplete, with holes, which correspond to the darker regions in the image and leave portions of the HOPG electrode surface uncovered.

The ODN sequence hybridizes with its complementary sequence via hydrogen bonding to form double helical strands and the ODN hybridization efficiency is influenced by the accessibility of the ODN target to the ODN probe immobilized on the electrode surface. In order to have the maximum accessibility of the target molecules for hybridization, the probe molecules should ideally be connected with the electrode surface only at one point, should be perpendicularly oriented to the electrode surface, and with the sequences sufficiently separated one from another. In the MAC Mode 

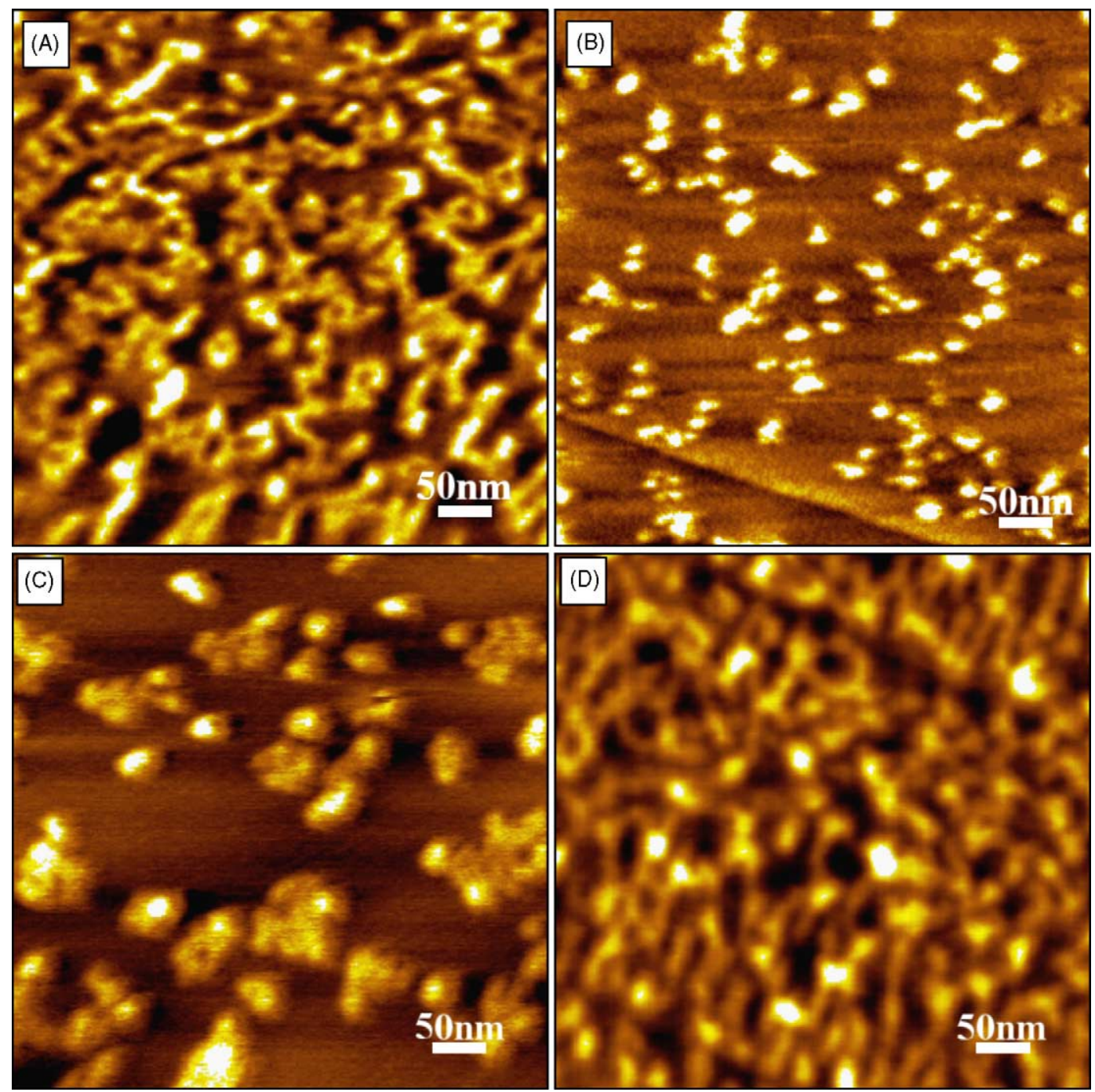

Fig. 1. MAC Mode AFM topographical images in air of the ODN sequences on the HOPG electrode surface. Immobilization of ODNs was performed by free adsorption during $3 \mathrm{~min}$, in $\mathrm{pH} 4.50 .1 \mathrm{M}$ acetate buffer electrolyte from a $0.3 \mu \mathrm{M}$ solution of: (A) oligo(1), (B) oligo(2), (C) oligo(3), and (D) oligo(4).

AFM image, Fig. 1A, an ODN film formed by many cross-over and superposed molecules is seen, which leads to restricted mobility of the bases bound to the electrode and prevents many ODN sequences undergoing hybridization, consequently reducing the hybridization efficiency.

The non-specific adsorption of the target ODN sequences represents another critical issue in the development of a DNA electrochemical biosensor. Ideally, the probe ODN must be sufficiently densely packed to prevent non-specific adsorption of the target probes in between the probe molecules. Additionally, as observed in the AFM images, Fig. 1A, due to the extensive existence of pores in the oligo(1) film, large areas of the HOPG surface were not covered. The fact that the electrode surface is not completely covered enables non-specific adsorption of other ODN sequences from the bulk solution. For this reason the free adsorption of oligo(2), oligo(3) and oligo(4), which are the complementary and non-complementary ODN sequences, was investigated.
Oligo(2), the complementary sequence of oligo(1), also adsorbs spontaneously at the HOPG electrode surface, Fig. 1B, but leads to a smaller degree of electrode surface coverage, compared to oligo(1), see Fig. 1A. The adsorbed molecules appeared in the AFM image as small bullets. The r.m.s. roughness of the HOPG surface covered by oligo(2) molecules was $0.18 \mathrm{~nm}$.

Performing free adsorption of oligo(3), a non-complementary sequence of oligo(1), the adsorption of the molecules in larger, broad groups of rods was observed, Fig. 1C. The molecules left much of the HOPG surface uncovered, and the value of the r.m.s. roughness was $0.52 \mathrm{~nm}$.

The topography after free adsorption at the HOPG surface of oligo(4), another non-complementary sequence of oligo(1), is seen in Fig. 1D. The ODN film had the aspect of an incomplete network, in the form of coiled fibres, which join together in end-to-end aggregates leaving parts of the HOPG surface uncovered. The oligo(4) film showed a r.m.s. roughness of $0.61 \mathrm{~nm}$. 
The MAC Mode AFM images show that both the complementary and the non-complementary ODN sequences adsorb on the carbon surface, which may lead to non-specific adsorption of these molecules on the areas of the electrode surface that are not covered by probe molecules. Consequently, in order to achieve a higher specificity and sensitivity of the DNA biosensors, non-specific adsorption of the target ODN molecule must be minimized.

A second immobilization method was used to attach the probe ODN molecules to the electrode surface, as described in the Section 2. This consisted in the adsorption of the probe sequence onto an ODN-modified electrode. The modification of the electrode surface was achieved by first applying a potential of $+1.0 \mathrm{~V}$ versus $\mathrm{AgQRE}$, for $3 \mathrm{~min}$, to the HOPG electrode immersed in a solution of oligo(1), the probe sequence.

This electrochemical adsorption procedure influenced the process of adsorption and growth of the oligo(1) film on the HOPG electrode. AFM images show the HOPG surface covered by a uniform, strong and compact ODN probe film, Fig. 2A-C. The electrode was almost completely covered by the ODN layer, with only a few pores. The topographical images enable distinction between two different areas on the film. Large zones look extremely smooth, with molecules condensed in a highly packed fashion with a high degree of compactness, while other parts of the ODN layer were much rougher, with highly packed ODN nuclei of many different sizes, from 1 to $3 \mathrm{~nm}$ height, and 10 to $40 \mathrm{~nm}$ diameter measured at half height, Fig. $2 \mathrm{C}$ and D. The calculated r.m.s. roughness of the entire oligo(1) probe film formed on the HOPG electrode and measured in the image from Fig. $2 \mathrm{C}$ was $0.78 \mathrm{~nm}$, demonstrating a much rougher topography compared with the oligo(1) probe film obtained by free adsorption, Fig. 1A.

The applied potential of $+1.0 \mathrm{~V}$ versus AgQRE, enhanced the stability of the oligo(1) molecules on the electrode sur-
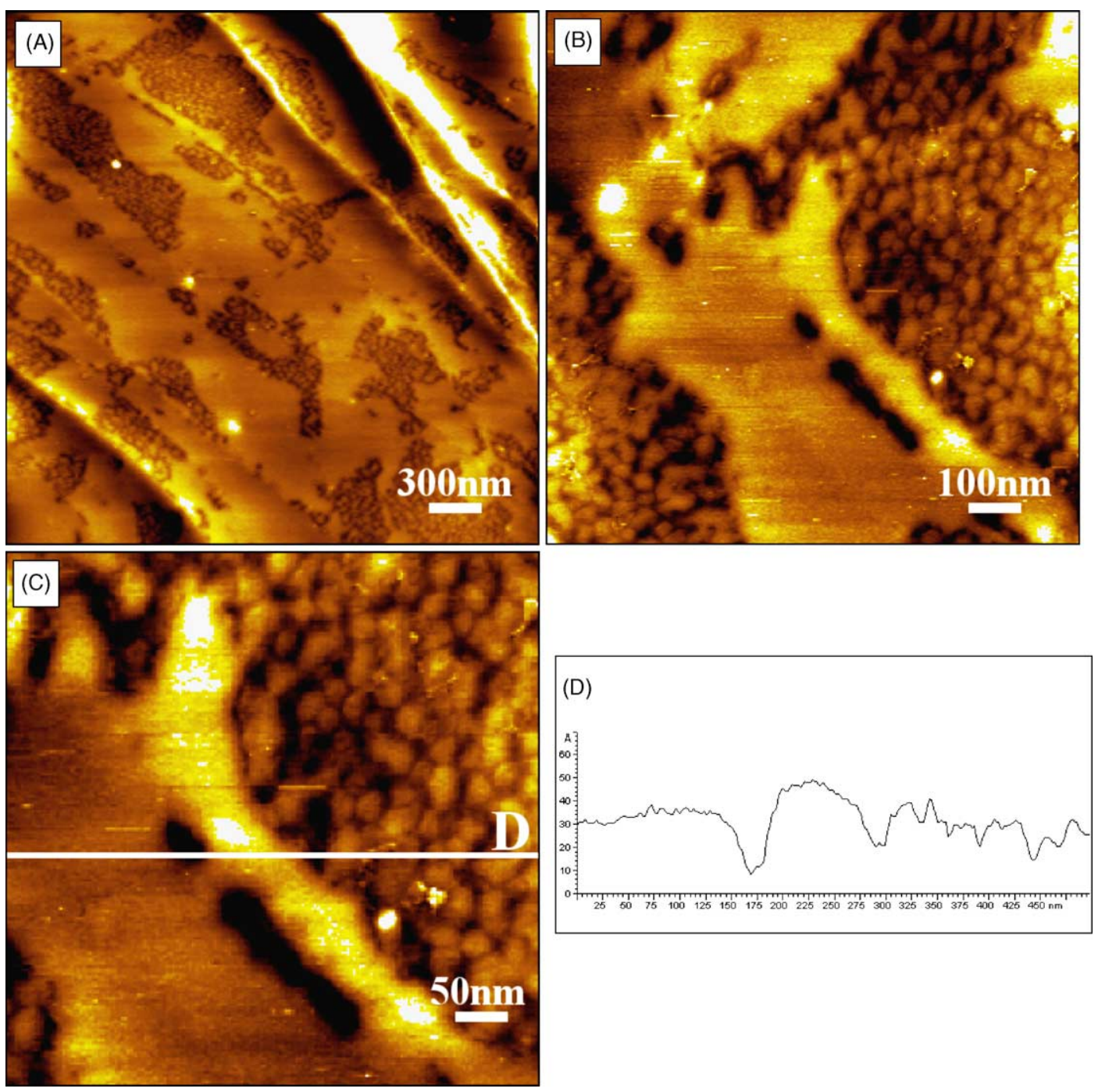

Fig. 2. (A-C) MAC Mode AFM topographical images in air of the ODN-modified HOPG electrode surface. Immobilization of the oligo(1) oligonucleotide was performed by applying a potential of $+1.0 \mathrm{~V}$ vs. AgQRE, for $3 \mathrm{~min}$, to the HOPG electrode immersed into $0.3 \mu \mathrm{M}$ oligo(1) solution, in pH 4.5 $0.1 \mathrm{M}$ acetate buffer electrolyte. (D) Cross-section profile through the white line in the image (C). 
face, due to stronger electrostatic interactions between the positive electrode and the negative sugar-phosphate DNA backbone. However, during the ODN-modification procedure, the very high applied potential also caused oxidation of guanine and adenine bases on the HOPG electrode surface. The oligo(1) probe molecules adsorbed on the HOPG electrode were therefore modified and the sequences contain the guanine and adenine oxidation products, and this gave rise to highly packed layers.

These two-dimensional layers completely covering the HOPG electrode form a biomaterial matrix, the ODNmodified electrode, onto which surface the oligo(1) probe molecules were subsequently attached. This ODN-modified electrode architecture may prevent further non-specific adsorption of target ODN molecules on the electrode surface. Hybridization electrochemical studies were therefore performed with this electrode.

\subsection{Voltammetry in incubated solutions}

The hybridization experiments were carried out in incubated solutions containing the two complementary ODNs. In Fig. 3A are presented the DP voltammograms recorded in buffer containing $0.3 \mu \mathrm{M}$ oligo(1), Fig. 3A (full line), and in a mixture of $0.3 \mu \mathrm{M}$ oligo(1) incubated for different periods with $0.3 \mu \mathrm{M}$ complementary oligo(2), Fig. 3A (dotted lines). Between each measurement the electrode surface was polished. Both guanosine and adenosine oxidation peaks gradually decreased in size with the incubation time and constant values were only obtained after $24 \mathrm{~h}$ of hybridization in solution.

At the same time, control experiments were carried out where $0.3 \mu \mathrm{M}$ oligo(1) was incubated for different time intervals with $0.3 \mu \mathrm{M}$ non-complementary oligo(5) or oligo(7). The DP voltammograms obtained after $24 \mathrm{~h}$ incubation of oligo(1) with oligo(5), Fig. 3B (dotted line), showed an increase of both guanosine and adenosine peaks when compared with the voltammogram obtained only in oligo(1), Fig. 3B (full line). The control oligo(5) sequence contains purinic bases, therefore the overall concentration of guanosine and adenosine increases and bigger peaks were obtained. On the other hand, DP voltammograms obtained after $24 \mathrm{~h}$ of incubation of oligo(1) with oligo(7), Fig. 3B (dashed line), showed practically no difference in the amplitude of the oxidation peaks of the target oligo(1). In this case, the control ODN sequence contains only pyrimidinic bases and thymine and cytosine oxidation is not detectable (Oliveira Brett and Matysik, 1997).

This experiment gives information on the possibility of electrochemical detection of an ODN sequence hybridization to a complementary sequence, and distinguishing between non-complementary sequences, only purines or pyrimidines, when present in solution. However, the limitations of such an experiment are obvious in terms of time and reagent consumption compared with using a DNA-biosensor (Oliveira Brett, 2004).

The immobilization of a known ODN sequence on a biosensor surface followed by hybridization in a solution containing a complementary sequence and transduction in pure electrolyte presents greater advantages. However, attention has to be paid to the probe immobilization conditions, in order to reduce the contribution of non-specific adsorbed ODN sequences (Wang, 2000). Indeed, a too high density of immobilized strands at the surface may not permit the complementary strands to hybridize, due to steric hindrance (Marquette et al., 2002). On the other hand, a low strand density at the surface may not be sufficient to generate electrochemical signals.
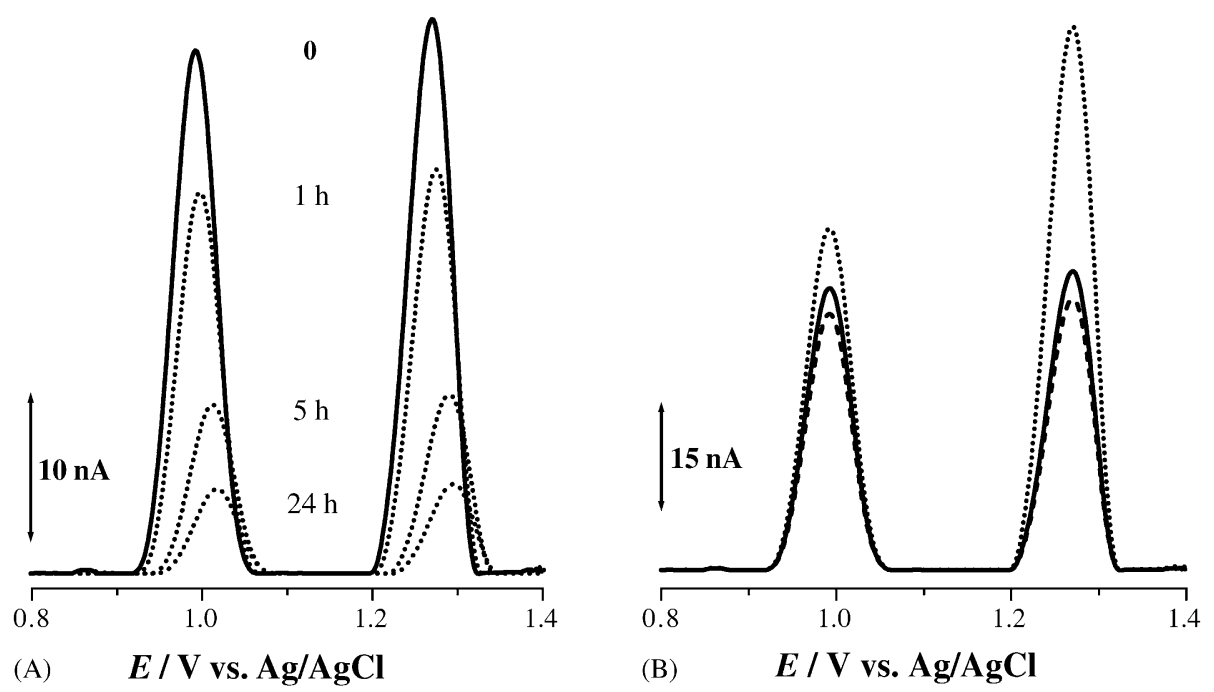

Fig. 3. DP voltammograms in $\mathrm{pH} 4.50 .1 \mathrm{M}$ acetate buffer containing: (-) $0.3 \mu \mathrm{M}$ oligo(1) before and after: (A) (....) different hybridization periods in solution with $0.3 \mu \mathrm{M}$ complementary oligo(2); (B) $24 \mathrm{~h}$ incubation in solution with $0.3 \mu \mathrm{M}$ non-complementary (....) oligo(5) or (...-) oligo(7). Scan rate $5 \mathrm{mV} \mathrm{s}^{-1}$, pulse amplitude $50 \mathrm{mV}$, pulse width $70 \mathrm{~ms}$. 


\subsection{Voltammetry of adsorbed oligo(1)}

The influence of electrode pretreatment on the oxidation peaks of immobilized oligo(1) was evaluated. Three different electrode surface conditioning procedures were investigated in order to optimize the ODN immobilization conditions. The first consisted in recording several DP voltammograms with a freshly polished electrode surface in $\mathrm{pH} 4.50 .1 \mathrm{M}$ acetate buffer until a stable base line was obtained. The second consisted in the application to a freshly polished electrode of a potential of $+1.4 \mathrm{~V}$ during 2 min in supporting electrolyte. In the third procedure, a freshly polished glassy carbon surface was used. After each pretreatment, the electrode was kept for $3 \mathrm{~min}$ in $0.3 \mu \mathrm{M}$ oligo(1) allowing free adsorption to occur. Then, the electrode was washed with ultrapure water and transferred to buffer where a DP voltammogram was recorded. The three voltammograms were compared. Very reproducible results and higher oxidation peaks for guanosine and adenosine were obtained at a freshly polished electrode surface without any previous conditioning. Therefore this procedure was used in the following experiments.

The effect of oligo(1) concentration and immobilization period on the guanosine and adenosine DP voltammetric peak currents was studied. Different probe concentrations ranging between 0.1 and $1.2 \mu \mathrm{M}$ were assessed in connection with $10 \mathrm{~s}$ to $10 \mathrm{~min}$ free adsorption time. After oligo(1) immobilization, the electrode was rinsed with acetate buffer and placed in supporting electrolyte where the DP voltammogram was recorded.

In Fig. 4A plots of guanosine and adenosine peak currents obtained after free adsorption of oligo(1) for $3 \mathrm{~min}$ in solutions of different concentration are shown. A linear increase of the peak currents with oligo(1) concentration varying between 0.1 and $0.5 \mu \mathrm{M}$ was found. For higher concentrations saturation occurred and constant peak currents of about $50 \mathrm{nA}$ for guanosine and $75 \mathrm{nA}$ for adenosine oxidation were reached.
The effect of adsorption time on the oligo(1) peak currents is presented in Fig. 4B. Free adsorption from a solution containing $0.3 \mu \mathrm{M}$ oligo(1) followed by detection in buffer showed a progressive increase of the peak currents over the first $3 \mathrm{~min}$ of adsorption. After that the surface became saturated and a maximum of $25 \mathrm{nA}$ for guanosine and $32 \mathrm{nA}$ for adenosine oxidation was obtained.

From these results, the optimized experimental conditions were $3 \mathrm{~min}$ of free adsorption in a $0.3 \mu \mathrm{M}$ solution of oligo(1). They ensured a good coverage of the electrode surface and were used for further characterization of the hybridization experiments carried out on a freshly polished electrode surface.

\subsection{Voltammetric studies of hybridization on a glassy carbon electrode surface}

DP voltammograms of an oligo(1) probe immobilized electrode before and after immersion in solutions containing oligo(2) target or non-complementary oligo(5) or oligo(7) sequences were recorded in buffer, Fig. 5. After each medium exchange the electrode was rinsed with acetate buffer to ensure the removal of unbound molecules. Also, between each experiment the electrode surface was polished.

The oligo(1) probe immobilization at the glassy carbon surface was carried out by free adsorption during $3 \mathrm{~min}$ in a solution containing $0.3 \mu \mathrm{M}$ oligo(1). Before transferring to supporting electrolyte, where transduction was carried out, the probe immobilized electrode was rinsed with acetate buffer. The DP voltammogram obtained showed two well separated peaks due to oxidation of guanosine and adenosine present in the ODN chain, Fig. 5 (full line).

In another experiment, the oligo(1) probe immobilization was followed by dipping the probe immobilized electrode surface into oligo(2) target solution during $5 \mathrm{~min}$. Then, the electrode was rinsed with acetate buffer and transferred
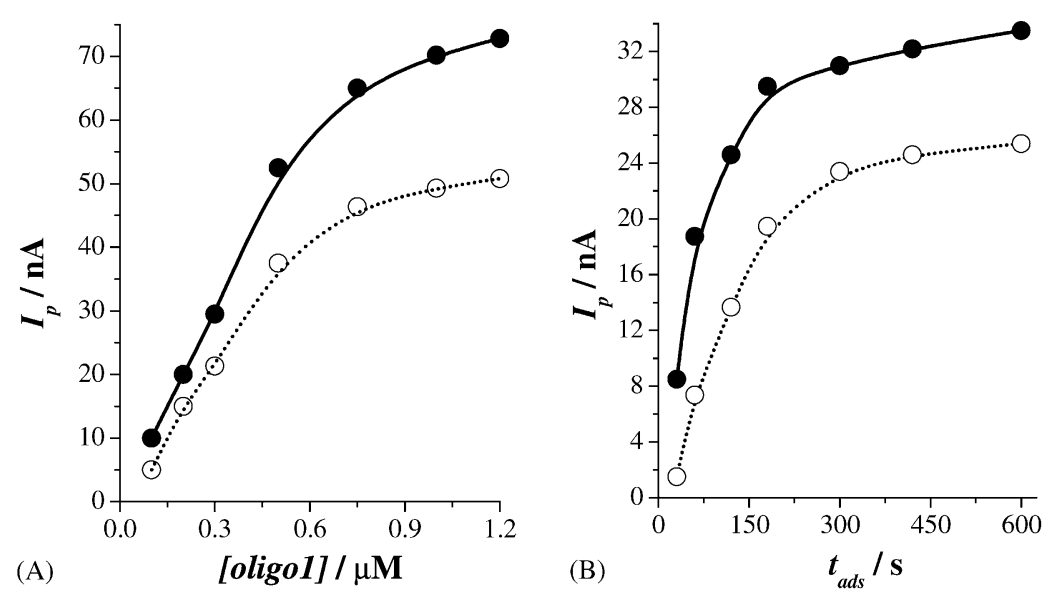

Fig. 4. Variation of $(\bigcirc)$ guanosine and $(-)$ adenosine DP oxidation peak currents, obtained in $\mathrm{pH} 4.50 .1 \mathrm{M}$ acetate buffer after free adsorption from a oligo(1) solution with: (A) concentration for $3 \mathrm{~min}$ adsorption time, and (B) adsorption time for a $0.3 \mu \mathrm{M}$ oligo(1) solution. 


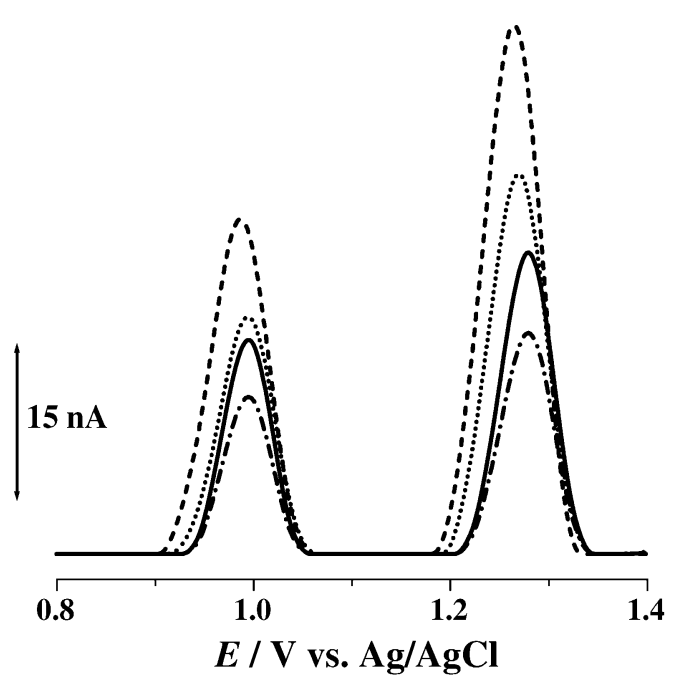

Fig. 5. DP voltammograms in $\mathrm{pH} 4.50 .1 \mathrm{M}$ acetate buffer obtained with a GCE after: (-) free adsorption for $3 \mathrm{~min}$ in $0.3 \mu \mathrm{M}$ oligo(1), incubation for $5 \mathrm{~min}$ of the oligo(1) immobilized at the electrode surface with the (....) complementary target oligo(2), control experiments for $5 \mathrm{~min}$ in complementary (-.-) oligo(5) or (_.-) oligo(7). Scan rate $5 \mathrm{mV} \mathrm{s}^{-1}$, pulse amplitude $50 \mathrm{mV}$, pulse width $70 \mathrm{~ms}$.

to pure electrolyte, where DP voltammetry was performed, Fig. 5 (dotted line). An increase of both guanosine and adenosine peak heights when compared with the previously obtained voltammogram was observed.

During the hybridization reaction hydrogen bonds are formed between complementary sequences leading to a duplex, inside which it is more difficult to oxidize the bases. For this reason, a decrease of guanosine and adenosine peaks should occur after hybridization. However, as shown by the MAC Mode AFM results, ODN molecules freely adsorb at the electrode surface in a network and regions of the electrode surface are exposed to the solution, Fig. 1. Therefore, target molecules present in solution can hybridize and can also adsorb at the probe-uncovered electrode regions leading to an increase of the overall guanosine and adenosine concentration on the electrode surface.

In order to evaluate the effect of non-specific adsorbed ODNs, control experiments were performed. The oligo(1) probe-coated electrode was placed for $5 \mathrm{~min}$ in a solution containing oligo(5) non-complementary strands. In Fig. 5 (dashed line), the DP voltammogram obtained after the control experiment in oligo(5) showed that both guanosine and adenosine peaks increased when compared with the DP voltammogram obtained after immobilization of oligo(1). Since no hybridization could have occurred during control experiments the increase of the signals is due to non-specific adsorbed oligo(5) molecules, the sequence of which contains only guanine and adenine bases.

In another control experiment the electrode with immobilized oligo(1) probe was held for $5 \mathrm{~min}$ in a solution containing non-complementary oligo(7). In this case the control ODN has only pyrimidinic bases and no effect upon the probe oxidation peaks is expected. After transferring to

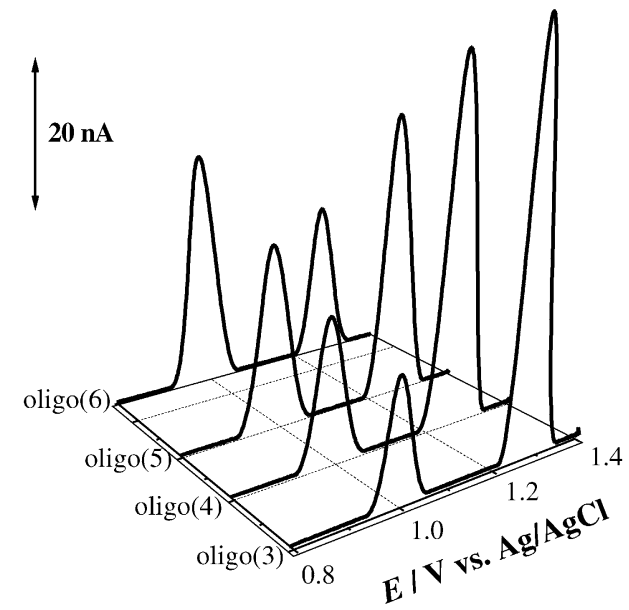

Fig. 6. 3D plot of DP voltammograms obtained after control experiments in $0.3 \mu \mathrm{M}$ non-complementary solutions of oligo(3), oligo(4), oligo(5) and oligo(6).

buffer the recorded DP voltammogram showed a different feature, Fig. 5 (dashed and dotted lines). Both guanosine and adenosine peak currents decreased when compared with the voltammogram obtained after immobilization of oligo(1). An explanation for this effect could be the increase of the overall surface negative charge during non-specific accumulation of non-complementary ODNs that induces a barrier to electron transfer.

Other control experiments were carried out using oligo(3), oligo(4) and oligo(6), each one presenting a different number of guanine residues. In each experiment were used 10-mer ODN sequences and the number of adenines in a given nucleotide chain was inversely proportional to the number of guanine residues. Therefore, a relationship of inverse proportionality between guanosine and adenosine oxidation peaks was expected.

In Fig. 6 is presented a three-dimensional plot of the voltammograms obtained as a function of the number of guanine residues in the ODN chain. A strong dependence between the number of guanine and adenine residues in the ODN chain and the peak currents obtained for the oxidation of guanosine and adenosine was observed. These experiments were repeated several times and good reproducibility was obtained.

The voltammetric experiments presented showed that during the hybridization reaction on a bare GCE surface, non-specific adsorption occurred that significantly influenced the results. On another hand, the control experiments have shown that the non-specific adsorption is strongly dependent on the nature (sequence) of the involved ODN.

\subsection{Voltammetric studies of hybridization on an ODN-modified electrode surface}

In order to reduce the contribution from non-specific adsorbed ODN sequences during hybridization, experiments 

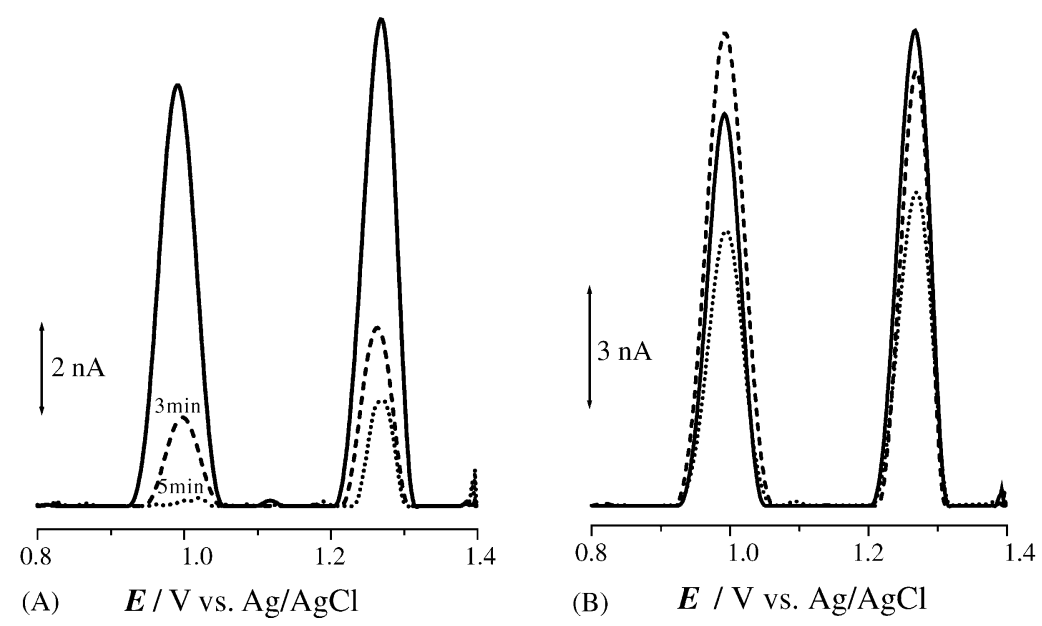

Fig. 7. DP voltammograms in pH 4.5 $0.1 \mathrm{M}$ acetate buffer obtained with an ODN-modified GCE after: (A) immobilization of (_) oligo(1) and hybridization for (...) $3 \mathrm{~min}$ and (....) $5 \mathrm{~min}$ in $0.3 \mu \mathrm{M}$ oligo(2) and (B) control experiments for $5 \mathrm{~min}$ in $0.3 \mu \mathrm{M}$ : (_) oligo(5), (_..) oligo(6) and (....) oligo(7). Scan rate $5 \mathrm{mV} \mathrm{s}^{-1}$, pulse amplitude $50 \mathrm{mV}$, pulse width $70 \mathrm{~ms}$.

using an ODN modification of the GCE surface were undertaken.

The GCE was immersed in a $0.3 \mu \mathrm{M}$ oligo(1)/pH 4.5 $0.1 \mathrm{M}$ acetate buffer solution, and the surface was modified by applying a potential of $+1.4 \mathrm{~V}$ during $2 \mathrm{~min}$. During this period the oligo(1) present in the solution adsorbed on the electrode surface but at the same time the purinic bases were oxidized. It was shown by the MAC Mode AFM images (Fig. 2), that the adsorption of oxidised ODN sequences forms a complex and compact pattern, which may act as a matrix for further immobilization of oligo(1) probe nucleotides. The adsorbed oxidized ODN bases may not distinguish between a complementary and a non-complementary strand. Also, during modification an overall increase of the negative charge at the electrode surface, caused by the strong adsorption of the negatively charged oxidised ODN sequence, will prevent further non-specific adsorption, due to electrostatic repulsion.

This ODN-modified GCE was immersed into oligo(1) probe. DP voltammograms of the oligo(1) probe immobilized onto the ODN-modified GCE before, Fig. 7A (full line), and after, Fig. 7A (dashed line), exposure to solutions containing target oligo(2) were recorded in buffer. After each medium exchange the electrode was thoroughly washed with acetate buffer to assure the removal of unbounded molecules. Also, between each experiment the electrode surface was polished.

The DP voltammogram obtained of the oligo(1) probe immobilized onto the ODN-modified GCE, Fig. 7A (full line), showed two peaks due to the oxidation of guanosine and adenosine present in the oligo(1) chain.

In the next experiment, the oligo(1) probe immobilization on the ODN-modified GCE was followed by dipping the electrode into oligo(2) target solution during $3 \mathrm{~min}$ to allow hybridization to occur, Fig. 7A (dashed line). A several fold decrease of both guanosine and adenosine peaks is observed when compared with the voltammogram obtained after oligo(1) probe immobilization. Longer hybridization periods led to the complete disappearance of guanosine oxidation peak and a big decrease in the adenosine oxidation signal. This was observed in a another experiment when the oligo(1) probe immobilization on the ODN-modified GCE was followed by dipping the modified surface into oligo(2) target solution during $5 \mathrm{~min}$ to allow the hybridization to occur, Fig. 7A (dotted line).

Control experiments were performed in which the ODN-modified GCE with the oligo(1) probe immobilized was held for $5 \mathrm{~min}$ in solutions each containing a different non-complementary ODN sequence. After transferring to buffer, DP voltammograms were recorded. Small fluctuations of guanosine and adenosine oxidation peaks were observed after the ODN-modified GCE with the immobilized oligo(1) probe was held in oligo(5), Fig. 7B (full line). Also, control experiments in oligo(3) and oligo(4) were carried out and the voltammograms obtained (not shown) show small variations of the peaks current if compared with the voltammogram obtained after probe immobilization.

A different situation was encountered when control experiments were carried out using ODN sequences containing pyrimidinic residues. As expected, a small increase in the guanosine peak was observed during the control experiment with oligo(6), which contains seven guanines and three cytosines, see Fig. 7B (dashed line), whereas a decrease of adenosine current is seen compared with the peaks obtained after oligo(1) probe immobilization. On the other hand, the voltammogram recorded after the control experiment with oligo(7), which contains only pyrimidines, Fig. 7B (dotted line), showed a different feature as both guanosine and adenosine peak currents decreased when compared with the voltammogram obtained after oligo(1) probe immobilization. This is explained by the adsorption of oligo(7) that causes a change in the orientation of oligo(1) immobilized on the ODN-modified electrode surface. 
The voltammetric hybridization experiments on an ODN-modified GCE showed that after hybridization a decrease in the guanosine and adenosine oxidation currents occurs, as expected. It was shown that non-specific adsorption is considerably reduced when using an ODN-modified GCE. Control experiments revealed a dependence of the non-specific adsorption on the ODN nature as different variation of the oxidation currents where observed depending on the ODN sequence. This effect could not be fully characterized using only voltammetric experiments and therefore more information should be sought concerning the modified electrode surface interfacial region.

\subsection{EIS characterization of the hybridization at ODN-modified electrode surface}

Electrochemical impedance spectroscopy (EIS) measurements were undertaken in order to characterize the electrode surface after modification, immobilization, hybridization and control experiments.

In Fig. 8 is presented the complex plane plot representation of the impedance spectra recorded at a ODN-modified GCE after oligo(1) probe immobilization, hybridization and control experiments. All measurements were performed at an applied potential of $+0.5 \mathrm{~V}$. Sequentially recorded spectra after the immobilization procedure show no different features proving that no detectable desorption of the ODN strands from the electrode surface took place during impedance measurements.

An almost pure capacitive behaviour was observed after immobilization of oligo(1), Fig. 8. Following the hybridization procedure described in Section 2.4 an increase in the impedance was detected, corresponding to a decrease of the interfacial capacitance, since in these cases the impedance follows a relationship of inverse proportionality with the capacitance of the system.

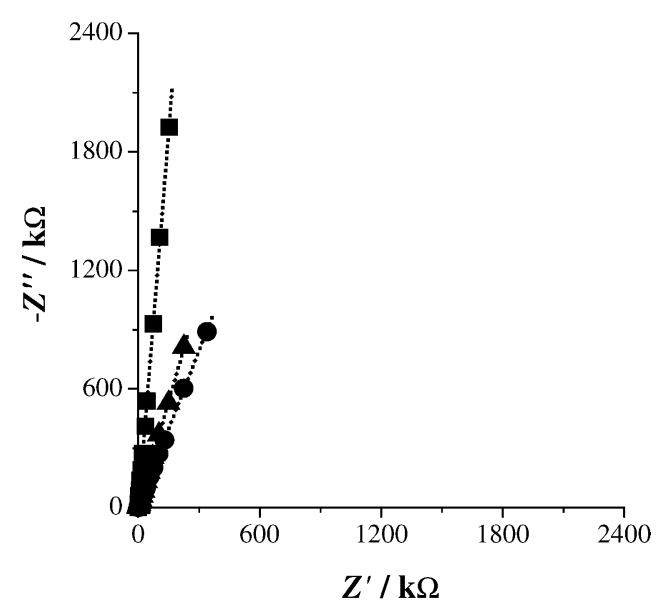

Fig. 8. Complex plane impedance spectra in $\mathrm{pH} 4.50 .1 \mathrm{M}$ acetate buffer at $+0.5 \mathrm{~V}$, obtained after: (-) ODN-modification of GCE and immobilization of oligo(1), ( $\mathbf{\square})$ hybridization with oligo(2) and ( $\mathbf{\Delta})$ control experiments with oligo(5).
Also, control experiments have been carried out with oligo(5). The impedance spectrum obtained for the control is observed as being intermediate between that recorded for oligo(1)-immobilized at the ODN-modified GCE and the impedance spectrum obtained after hybridization.

Fitting of the spectra was done using an equivalent circuit composed of a RCPE parallel combination in series with the cell resistance $R_{\Omega}=540 \Omega$, Scheme 1 . Resistance $R$ represents the charge transfer resistance, $R_{\mathrm{ct}}$, and the capacitance was represented by a constant phase element, $Q$, in order to take into account the frequency dispersion behaviour due to roughness and porosity normally found at solid electrodes (Brett et al., 1999; Oliveira Brett et al., 2002). Fitting of the spectra was very good using this circuit for all experimental spectra.

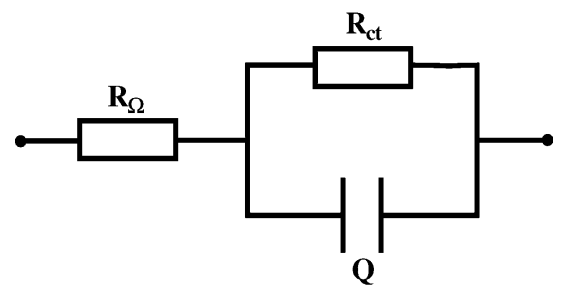

Scheme 1. Equivalent electrical circuit used to fit the impedance data obtained after immobilization, hybridization and control experiments at an ODN-modified GCE.

A decrease (one order of magnitude) in the capacitance was observed after the hybridization experiment, see Table 1, when compared with the spectra obtained after immobilization or control experiments. A smaller capacitance value was obtained also after the control experiment when compared with the spectra obtained with immobilized target and this is due to non-specific adsorption of non-complementary strands. On the other hand, the $R_{\mathrm{ct}}$ value obtained after hybridization is significantly lower (almost two orders of magnitude) than after immobilization and control experiments. Also, an increase in roughness $(n \approx 0.7)$ was observed after the hybridization experiment.

The capacitive behaviour of the system can be more conveniently represented using the capacitance data obtained from the impedance spectra. Changes taking place in the double layer capacitance during modification, immobilization, hybridization and control experiments are plotted as a function of the time period of the applied ac perturbation, Fig. 9. Changes of the capacitance could be easily followed for long time periods of perturbation. In this case modifications in the double layer are taking place slowly and the

Table 1

Results obtained after fitting the impedance spectra with the equivalent circuit

\begin{tabular}{lccl}
\hline & $R_{\mathrm{ct}}\left(\mathrm{k} \Omega \mathrm{cm}^{2}\right)$ & $C\left(\mu \mathrm{F} \mathrm{cm}{ }^{-2}\right)$ & $n$ \\
\hline Immobilization & 176 & 12.5 & 0.84 \\
Hybridization & 13.7 & 0.68 & 0.74 \\
Control-oligo(5) & 173 & 9.66 & 0.82 \\
\hline
\end{tabular}




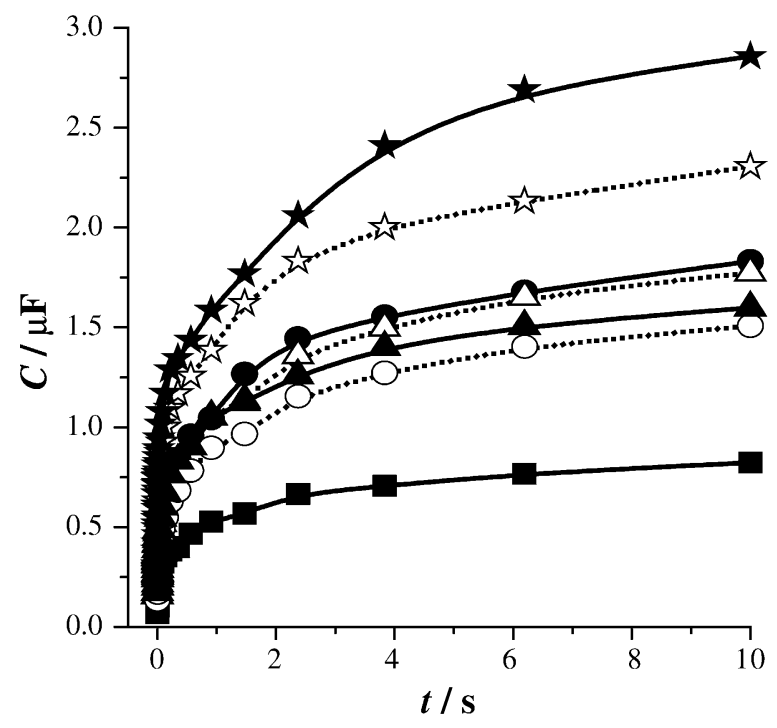

Fig. 9. Capacitance data from impedance spectra in $\mathrm{pH} 4.50 .1 \mathrm{M}$ acetate buffer at $+0.5 \mathrm{~V}$ obtained after: $(\star)$ pretreatment of bare GCE for $2 \mathrm{~min}$

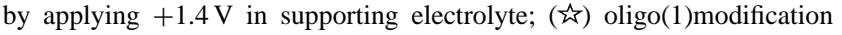
of GCE; immobilization of oligo (1) for (O) $3 \mathrm{~min}$ and (O) $5 \mathrm{~min}$, ( $\square$ ) hybridization with oligo(2) for $5 \mathrm{~min}$; control experiments for $5 \mathrm{~min}$ in $(\triangle)$ oligo(5) and ( $\mathbf{\Delta})$ oligo(7).

initial conformation (before the ac perturbation was applied) is reached before the system is again disturbed.

The capacitance variation with frequency of the glassy carbon electrode double layer before and after electrode modification is shown in Fig. 9. Because the electrode surface modification was carried out by applying $+1.4 \mathrm{~V}$ during 2 min in oligo(1), impedance spectra of a clean GCE was also recorded after pretreatment for $2 \mathrm{~min}$ at $+1.4 \mathrm{~V}$ in acetate buffer. A decrease of the GCE capacitance was observed after surface modification with oligo(1). Furthermore, the ODN-modified GCE was used to study the capacitive changes after immobilization, hybridization and control experiments.

Fig. 9 shows the spectra obtained after free adsorption for 3 and $5 \mathrm{~min}$ in a solution containing $0.3 \mu \mathrm{M}$ oligo(1). It is observed that probe immobilization leads to a decrease in the capacitance when compared with that of the ODN-modified GCE. Lower values of the capacitance were observed if the adsorption time was increased.

Following the hybridization procedure described in Section 2.4 a large drop in the capacitance was observed. As the hybridization reaction proceeds both the dielectric thickness and the medium permitivity change such that the capacity value is decreasing (Wei et al., 2003). It was also observed that, due to a greater rigidity of the double helix form, the difference of capacitances measured at high and small frequency is not as obvious as in the case of the single stranded form.

Non-specific adsorption as a contributor to the overall impedance change was addressed in control experiments. When the control experiments were carried out in oligo(7), that contains only pyrimidinic bases, a drop in the capacitance of the double layer formed by immobilized probe at the ODN-modified GCE surface was observed. Since no hybridization could have occurred during this experiment the capacitance decrease is attributed to the non-specific adsorption of the non-complementary ODN sequence. The same situation was encountered when control experiments were performed with oligo(6). On the other hand, when using oligo(5) was the ODN sequence used as a control the spectrum obtained is close to that recorded after the oligo(1) immobilization procedure. All other non-complementary ODNs were tested in control experiments and the spectra recorded confirmed the behaviour obtained with oligo(5).

The impedance spectra demonstrate that when hybridization experiments are carried out at an ODN-modified GCE non-specific adsorption is considerably reduced. However, both selective and non-selective binding to immobilized ODN layers depended on the local environment of the immobilized ODN.

\section{Conclusions}

The results presented in this paper demonstrate the importance of characterizing and understanding the adsorption process of ODN sequences on the carbon electrode surface, for a correct evaluation of all the factors influencing the response of the DNA electrochemical biosensors. The MAC Mode AFM images revealed that all probe, complementary and non-complementary ODN sequences adsorb spontaneously on the electrode surface. The images illustrated the existence of pores on the adsorbed layers, which left uncovered large parts of the electrode surface. This aspect must be carefully considered when using DNA electrochemical biosensors for the detection of DNA hybridization processes, due to the non-specific adsorption of molecules on the uncovered areas.

Hybridization experiments carried out in solution show that the recognition process between two complementary oligonucleotides occurs slowly and can be followed by DP voltammetry. The data obtained after immobilization of an oligonucleotide at the electrode surface show different features. Increased guanosine and adenosine oxidation currents due to non-specific adsorbed complementary or non-complementary ODNs were obtained after hybridization and control experiments. Both selective and non-selective binding to immobilized ODN layers depend on the local environment of the immobilized ODN. The ODN modification of the carbon electrode surface improved the selective binding and reduced the adsorption of non-complementary strands, as observed in both electrochemical and AFM studies. The ODN that covered the modified carbon electrode surface acted as a biomaterial matrix for further attachment of the probe ODN sequence and prevented the non-specific adsorption of target ODN molecules. 


\section{Acknowledgements}

Financial support from Fundação para a Ciência Tecnologia (FCT), Ph.D. Grant PRAXIS SFRH/BD/877/2000 (V.C.D.) and post-doctoral Grant SFRH/BPD/14425/2003 (A.-M.C.P.), POCTI (co-financed by the European Community Fund FEDER), ICEMS (Research Unit 103), and European Projects ERB-ICT15-CT960804 and HPRN-CT-200200186 are gratefully acknowledged.

\section{References}

Breslauer, K.J., Frank, R., Blöcker, H., Marky, L.A., 1986. Predicting DNA duplex stability from the base sequence. Proc. Natl. Acad. Sci. U.S.A. 83, 3746-3750.

Brett, C.M.A., Oliveira Brett, A.M., Serrano, S.H.P., 1999. An EIS study of DNA-modified electrodes. Electrochim. Acta 44, 4233-4239.

Chiti, G., Marrazza, G., Mascini, M., 2001. Electrochemical DNA biosensor for environmental monitoring. Anal. Chim. Acta 427, 155-164.

Ito, K., Hashimoto, K., Ishimori, Y., 1996. Quantitative analysis for solid-phase hybridization reaction and binding reaction of DNA binder to hybrids using a quartz crystal microbalance. Anal. Chim. Acta 327, 29-35.

Kleinjung, F., Bier, F.F., Warsinke, A., Scheller, F.W., 1997. Fibre-optic genosensor for specific determination of femtomolar DNA oligomers. Anal. Chim. Acta 350, 51-58.

Marquette, C.A., Lawrence, I., Polychronakos, C., Lawrence, M.F., 2002. Impedance based DNA chip for direct $T_{\mathrm{m}}$ measurements. Talanta 56 , 763-768.

Nicolini, C., Erokhin, V., Facci, P., Guerzoni, S., Ross, A., Paschkevitsch, P., 1997. Quartz balance DNA sensor. Biosens. Bioelectr. 12, 613-618.

Oliveira Brett, A.M., Matysik, F.M., 1997. Voltammetric and sonovoltammetric studies on the oxidation of thymine and cytosine at a glassy carbon electrode. J. Electroanal. Chem. 429, 95-99.

Oliveira Brett, A.M., Serrano, S.H.P., Gutz, I., La-Scalea, M.A., e Cruz, M.L., 1999. Studies on the mechanism of interaction of in situ produced nitroimidazole reduction derivatives with DNA using an electrochemical DNA-biosensor. In: Packer, L. (Ed.), Methods in Enzymology, vol. 300, Part B, pp. 314-321.

Oliveira Brett, A.M., Brett, C.M.A., Silva, L.A., 2002. An impedance study of the adsorption of nucleic acid bases at glassy carbon electrodes. Bioelectrochemistry 56, 33-35.

Oliveira Brett, A.M., Chiorcea, A.-M., 2003. Atomic force microscopy of DNA immobilized onto a highly oriented pyrolytic graphite electrode surface. Langmuir 19, 3830-3839.

Oliveira Brett, A.M., Chiorcea, A.-M., 2004. Atomic force microscopy characterization of an electrochemical DNA-biosensor. Bioelectrochemistry $63,229-232$.
Oliveira Brett, A.M., 2004. In: Gordon, L. (Ed.), DNA-based Biosensors, Comprehensive Analytical Chemistry, Biosensors and Modern Specific Analytical Techniques. Elsevier, Chapter 8.

Palecek, E., Fojta, M., Jelen, F., 2002. New approaches in the development of DNA sensor: hybridization and electrochemical detection of DNA and RNA at two different surfaces. Bioelectrochemistry 56, 8590.

Palecek, E., Fojta, M., Tomschik, M., Wang, J., 1998. Electrochemical biosensors for DNA hybridization and DNA damage. Biosens. Bioelectr. 13, 621-628.

Pedano, M.L., Rivas, G.A., 2003. Immobilization of DNA on glassy carbon electrodes for the development of affinity biosensors. Biosens. Bioelectr. 18, 269-277.

Piunno, P.A.E., Krull, U.J., Hudson, R.H.E., Damha, M.J., Cohen, H., 1995. Fiber-optic DNA sensor for fluorometric nucleic acid determination. Anal. Chem. 67, 2635-2643.

Pividori, M.I., Merkoçi, A., Alegret, S., 2000. Electrochemical genosensor design: immobilization of oligonucleotides onto transducer surface and detection methods. Biosens. Bioelectr. 15, 291-303.

Ratilainen, T., Holmen, A., Tuite, E., Haaima, G., Christensen, L., Nielsen, P.E., Norden, B., 1998. Hybridization of peptide nucleic acid. Biochemistry $37,12331-12342$.

Strasak, L., Dvorak, J., Hason, S., Vetterl, V., 2002. Electrochemical impedance spectroscopy of polynucleotides adsorption. Bioelectrochemistry $56,37-41$.

Su, H., Thompson, M., 1995. Kinetics of interfacial nucleic acid hybridization studied by acoustic network analysis. Biosens. Bioelectr. 10, 329-340.

Vetterl, V., Papadopoulos, N., Drazan, V., Strasak, L., Hason, S., Dvorak, J., 2000. Nucleic acid sensing by impedance measurements. Electrochim. Acta 45, 2961-2971.

Wang, J., 2000. From DNA biosensors to gene chips. Nucl. Acids Res. 28, 3011-3016.

Wang, J., 2002. Electrochemical nucleic acid biosensors. Anal. Chim. Acta 469, 63-71.

Wang, J., Xu, D., Erdem, A., Polsky, R., Salazar, M.A., 2002. Genomagnetic electrochemical assays of DNA hybridisation. Talanta 56, 931-938.

Waterson, J.H., Piunno, P.A.E., Krull, U.J., 2002a. Toward the optimization of an optical DNA sensor: control of selectivity coefficients and relative surface affinities. Anal. Chim. Acta 457, 29-38.

Waterson, J.H., Piunno, P.A.E., Krull, U.J., 2002b. Practical physical aspects of interfacial nucleic acid oligomer hybridization for biosensor design. Anal. Chim. Acta 469, 115-127.

Wei, F., Sun, B., Liao, W., Ouyang, J., Zhao, X.S., 2003. Achieving differentiation of single-base mutation through hairpin oligonucleotides and electric potential control. Biosens. Bioelectr. 18, 11491155 .

Yang, M., McGovem, M.E., Thompson, M., 1997. Genosensor technology and the detection of interfacial nucleic acid chemistry. Anal. Chim. Acta 346, 259-275. 